\section{Deuteron-gamma angular correlations}

from P. E. Hodgson

WHEN deuterons are inelastically scattered by nuclei they leave the target nucleus in an excited state, which usually decays by gamma emission. The angular correlation between the inelastically scattered deuteron and the subsequent deexcitation gamma ray can provide important information about the mechanism of the reaction.

An illustration of this has recently been provided by the work of Scheib, Hofmann and Vogler (Phys. Rev. Iett., 34, 1586; 1975 ) on the inelastic scattering of 10 $\mathrm{MeV}$ deuterons by ${ }^{24} \mathrm{Mg}$. The angular correlation was measured for gamma rays emitted in the reaction plane, and in this case the correlation function is given by

$$
\begin{aligned}
W\left(\boldsymbol{\varphi}_{\gamma}\right)=A & +B \sin ^{2}\left(\boldsymbol{\varphi}_{\gamma}-\boldsymbol{\varphi}_{1}\right)+ \\
& +C \sin ^{2} 2\left(\boldsymbol{\varphi}_{\gamma}-\boldsymbol{\varphi}_{2}\right)
\end{aligned}
$$

where $\varphi_{\gamma}$ is the angle between the beam direction and the angle of emission of the gamma ray, and $A, B, C, \varphi_{1}$ and $\varphi_{2}$ are constants whose values are obtained by fitting the experimental data. The constants $A, B$, and $C$, are related to products of the reaction substate amplitudes, which may be calculated from a detailed theory of the reaction. This makes the correlation function much more sensitive to the details of the reaction than is the differential cross section, which is given by the sum of the absolute squares of all the reaction amplitudes. The sensitivity allows the correlation function to tell us more about the details of the reaction mechanism than we could learn from the differential cross section alone.

This is strikingly shown by the ${ }^{24} \mathrm{Mg}$ results. The figure shows the differential cross section compared with calculations using two different models of the reaction. The upper curves show the results of distorted wave calculations with three different sets of distorting potentials and assuming that the reaction takes place in a single step from the ground state to the $2^{+}$excited state. The lower curves show similar calculations that also take into account the coupling to the $4^{+}$state, so that some of the excited $2^{+}$states are formed by first exciting the $4^{+}$state directly, followed by de-excitation to the $2^{+}$ state. All the calculations used the symmetric rotator model including spin-orbit interactions.

When comparing such theories with the experimental data we look for a good fit to the main forward peak, which is given by both theories and all potentials, together with a qualitative agreement with the rest of the cross section. The angle and width of the second peak are given quite well but its magnitude is somewhat underestimated by all calculations, rather more so by the theory including the two-step process than by the one-step theory. All these fits are acceptable, but do not distinguish between the two reaction mechanisms.

When we examine the deuteron-gamma correlation data the comparison is quite

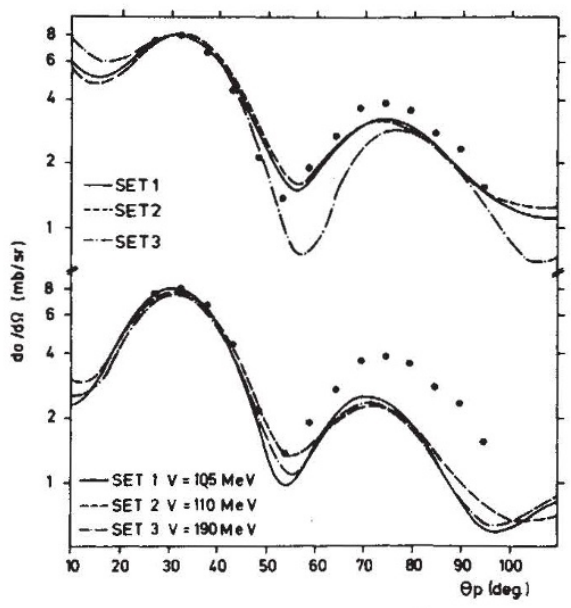

Differential cross section for the inelastic scattering of $10 \mathrm{MeV}$ deuterons by ${ }^{24} \mathrm{Mg}$ with excitation of the lowest $2^{+}$state compared with distorted wave calculations with three sets of distorting potentials using the one-step theory (upper curves) and the two-step theory (lower curves). $\mathrm{V}$ is the depth of the real part of the distorting potential. different. If the results for the parameter $C$ in the above expression for $W\left(\varphi_{\gamma}\right)$ are compared with the results of the six calculations already mentioned, the greater sensitivity of the correlation function is obvious. The three calculated curves for the one-step theory differ markedly and none fits the data, even qualitatively; however two of the calculations for the two-step theory are in good qualitative agreement with the data, and in particular give the deep minimum found around $55^{\circ}$. These two potentials are quite similar, and correspond to the deuteron potentials known to be physically realistic by calculations based on the nucleon-nucleus potential and using the folding model. The third potential for the two-step theory is physically unrealistic and also fails to give a good fit to the angular correlation data.

It is of course well known from many other studies that two-step processes must be taken into account in a detailed treatment of inelastic scattering, and this is confurmed by the correlation analysis. What is new is the striking way that it definitely excludes the one-step process as a sufficient explanation; this shows that the correlation analysis could be a powerful way of determining the reaction mechanism in cases where it is not already known.

\title{
Tunable alkali halide lasers
}

from John Walker

TUNABLE lasers, particularly dye lasers, now cover the whole of the visible spectrum, and are currently revolutionising optical spectroscopy. Unfortunately dye lasers do not operate in the near infrared, a region of great importance in molecular spectroscopy, pollution detection and fibre optic communications. Hence the significance of a recent paper by Mollenauer and Olson $(J$. appl. Phys., 46, 3109; 1975) who have demonstrated tunable laser action in potassium chloride, and have suggested that the wavelength range 0.9 to $3 \mu \mathrm{m}$ could be covered by suitable choice of alkali halide. These compounds, so important in our understanding of solid state physics and of point defects, have at last found a practical application.

Laser action over the range 2.5 to $2.9 \mu \mathrm{m}$ was achieved using the $\mathrm{F}_{\mathrm{A}}$ (II) centre in lithium-doped potassium chloride pumped by a krypton ion laser at $647.1 \mathrm{~nm}$. An input power of $40 \mathrm{~mW}$ was necessary when the $\mathrm{KCl}$ crystal was at $77 \mathrm{~K}$, and lasing could be induced at temperatures up to $200 \mathrm{~K}$.

The operation of the laser can be explained as follows. The $F_{A}(I I)$ centre consists of a halide vacancy (an F centre) trapped by an impurity metal ion-in this case lithium occupying a potassium lattice site. The centre is excited by the pump laser and then relaxes by rearrangement of the chloride ions around the vacancy. A population inversion is thereby achieved between the relaxed excited state and the unrelaxed ground state, which is emptied by further atomic rearrangement into the relaxed ground state. The system is therefore an ideal four-level laser.

Similar arguments apply to the F and the $F_{A}(I)$ centres, but in their case the excited state relaxation is very small, and there are competing de-excitation processes, making them unsuitable for laser action. However, two other potentially useful defects are the $\mathrm{F}_{2}{ }^{+}$centre (a pair of adjacent halide vacancies), and the $\mathrm{F}_{\mathrm{B}}(\mathrm{II})$ centre (similar to the $\mathrm{F}_{\mathrm{A}}(\mathrm{II})$, but with two impurity atoms). These centres should enable a total tuning range of 0.9 to $3 \mu \mathrm{m}$ to be achieved, and Mollenauer and Olson are currently trying to construct an $\mathrm{F}_{2}{ }^{+}$centre laser using $\mathrm{KCl}$.

The combination of simplicity, tunability, low pump power and potential frequency stability makes alkali halide lasers a promising prospect in the near infrared. 